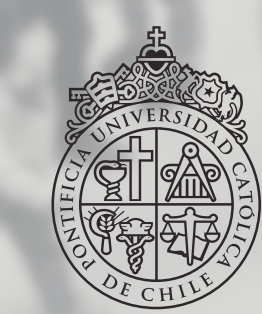

PONTIFICIA

UNIVERSIDAD

CATÓLICA

DE CHILE
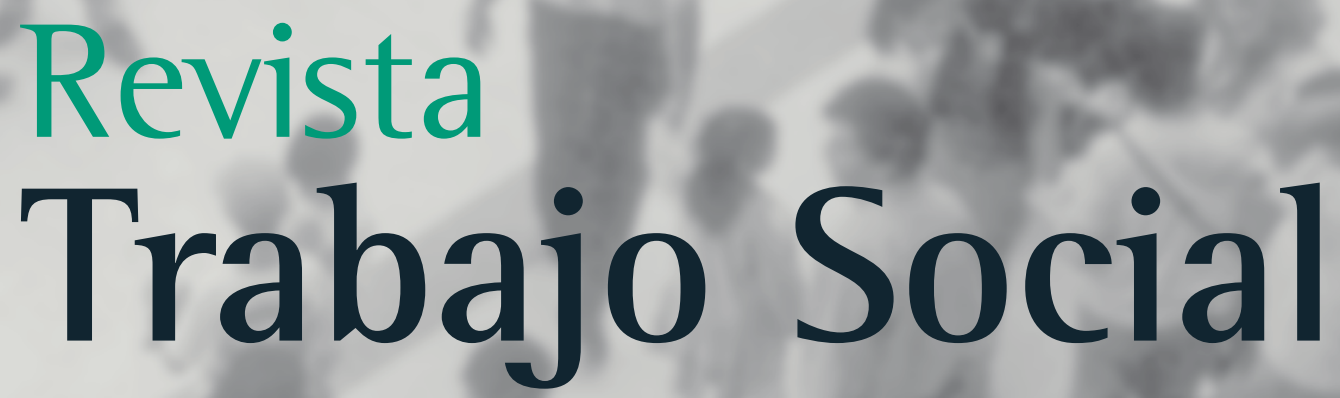

Intervención

socioambiental:

intersecciones del

Trabajo Social y la

institucionalidad

ambiental

Dr. Nelson Arellano-Escudero.

\section{Tensiones en la} Intervención Social:

(des)encuentros en la relación

Estado-ONG. Estudio de caso

sobre ONG que opera la política

social de infancia

Mauricio Sánchez Aliaga,

Rosa Villarroel Valdés.
Calidad de la participación infantil en la Política Local de Infancia Chilena

Daniela Díaz Bórquez,

Natalia Bozo Carrillo,

Nicolás Contreras SHats.

\section{Identidades Subyugadas}

entre Actores del Proceso

Penitenciario de Valparaíso

Paula Mercado Amin,

Fernanda Zavala Ossandón.

\section{Involucramiento} laboral

y disposición al cambio organizacional en el sector

de salud primaria

Carla Flores Figueroa,

LoRena MuÑoz MadRID,

Julia Jaramillo de Palacio. 



\title{
Involucramiento laboral y disposición al cambio organizacional en el sector de salud primaria
}

\section{Involvement Labor and Disposal at Organizational Change in Primary Healthcare}

\author{
Carla Flores Figueroa \\ Escuela de Trabajo Social, Universidad Santo Tomás. Email: carlaflores@santotomas.cl \\ LORENA Muñoz MadRID \\ Escuela de Trabajo Social, Universidad Santo Tomás. Email: lorenamunozma@santotomas.cl \\ Julia Jaramillo De Palacio \\ Escuela de Trabajo Social, Universidad Santo Tomás. Email: juliajaramillode@santotomas.cl
}

\begin{abstract}
Resumen
La presente investigación tiene por objeto determinar la incidencia que tienen los principales factores del involucramiento laboral en la adhesión al nuevo modelo de atención biopsicosocial en los centros de atención primaria de la comuna de Cerro Navia. Para esto se empleó una estrategia de investigación cuantitativa no experimental. Se aplicó una encuesta al 50\% de los funcionarios de los servicios de salud (187 personas), y luego se analizó la información a través de un proceso de análisis estadístico descriptivo bivariante y pruebas no paramétricas. A modo de conclusión, se determinó que existía una relación estadisticamente significativa entre las variables, es decir, el involucramiento laboral, la satisfacción y la percepción respecto al apoyo organizacional influyen positivamente en la aceptación del nuevo modelo de salud familiar por parte del personal de salud.

Palabras clave: Involucramiento laboral, atención primaria y modelo de salud familiar.

Abstract

This research determined the incidence of the Main Factors of labor involvement in the adherence of health personnel to the new model of biopsychosocial Care Centers Primary Care Cerro Navia. This paragraph employment strategy a non-experimental quantitative research and applied a survey, in 2014, 187 professional and nonprofessional randomly, corresponding to 50\% of the total population. Information Analysis took a corporal one through a process bivariate descriptive statistical analysis and nonparametric tests. To conclude, it did determine that there was a statistically significant relationship between the variables, $I E_{1}$ job involvement, satisfaction and perception of the organizational support positively influence acceptance of New Model of Family Health by staff Health.
\end{abstract}

Key words: Involvement job, primary health care and family health model.

\section{Antecedente}

Durante la dictadura militar predominó en Chile un modelo de atención eminentemente curativo, es decir, las políticas obviaron todos los factores sociales que podían influir en la salud de las personas y restringieron la participación de los pacientes, a pesar de que la situación epidemiológica y demográfica experimentaba cambios. De hecho, las enfermedades infecciosas y parasitarias que generaban el 32\% de las defunciones en 1950 (Instituto Nacional de
Estadísticas, 2012) habían sido sustituidas por las enfermedades al sistema circulatorio, que junto con los tumores malignos concentraban en 1990 el 44,5\% de las causas de las defunciones (Departamento de Estadísticas e Información de Salud, 2011). Además, la tasa de fecundidad había disminuido de 4,95 hijos por mujer en 1950 a 2,55 en 1990, y la esperanza de vida había aumentado de 54,8 años a 74, 3 en el mismo período (Véliz, 2012). 
Por otra parte, en la década de los '80 aparecieron una serie de estudios que desafiaron el modelo de atención biomédico centralizado en los hospitales, y que desacreditaron la idea de que más cuidado médico podía generar mejores resultados de salud. Específicamente, en Inglaterra se generó en 1980 el denominado "Informe Negro", que marcó un hito en la comprensión de la relación que existe entre los determinantes sociales y los resultados de la salud (Solar \& Irwin, 2010a). Por lo demás, rápidamente se fue generando evidencia consistente respecto a este fenómeno. Por ejemplo, también en Inglaterra, se demostró que una persona cuyos padres son profesionales puede llegar a vivir 5 años más que un hijo de un obrero no calificado; en España, se acreditó que mueren el doble de recién nacidos entre las familias rurales que entre las urbanas (Solar \& Irwin, 2010b); y en Chile era posible observar "una clara gradiente de prevalencias de hipertensión arterial, creciente en estratos con menor número de años de estudio, así, los niveles educacionales bajos presentaban una prevalencia de $51,1 \%$, mientras que los medio y altos de solo $22,8 \%$ y $16,7 \%$ respectivamente" (Ministerio de Salud de Chile, 2011, p. 58).

No obstante, cuando el país regresó a la democracia en 1990, el nuevo gobierno se encontró con un sistema de salud público indiscutiblemente desactualizado y en una condición de postración financiera que le impidieron hacerse cargo inmediatamente de los cambios epidemiológicos que se habían experimentado y de la evidencia que existía respecto a la influencia de los factores sociales en los resultados de la salud. Por esto, en un primer momento el Estado sólo pudo realizar importantes inversiones en infraestructura y recursos humanos, pero no intervenir en el modelo de atención.

En este contexto, una década después, todos los diagnósticos evidenciaban que el sistema de salud no respondía a las transformaciones epidemiológicas y que "la atención en salud seguía mostrando serias deficiencias en aspectos tales como la oportunidad de la prestación médica, el trato otorgado a los usuarios y la eficiencia en el uso de los recursos humanos" (Larrañaga, 2010). Por este motivo, en el año 2000 el Estado decidió impulsar una reforma de salud que relevara el rol del sistema de atención primaria, ya que era el nivel que en mayor medida recogía y contribuía a las transformaciones culturales que se necesitaban para abordar los cambios anteriormente expuestos. En este marco, en el 2004 se planteó un nuevo modelo de atención de salud con enfoque integral y familiar que se basa en el paradigma biopsicosocial que, sin desconocer la va- lidez del paradigma biomédico, ofrece una visión integral del proceso salud-enfermedad, incorporando otras dimensiones imprescindibles para el estudio y abordaje de la salud de las personas. A partir de ese entonces, se les ha exigido a los equipos de salud que observen los aspectos biológicos, psicológicos y sociales que inciden en la aparición de la enfermedad, pero que además pongan atención en las experiencias subjetivas de los pacientes. Las principales características de este modelo son:

- Familia y comunidad como unidad de atención: los equipos de salud no deben centrar su atención sólo en el paciente, sino que, además, su objeto de cuidado deber ser la familia y la comunidad en que éste vive.

- Atención centrada en el paciente: se debe explorar con el paciente tanto la enfermedad como la experiencia de padecerla y para ello se debe indagar en sus sentimientos, emociones e historia (Stewart, 2003).

- Continuidad de la atención: los profesionales de la salud "deben mantener una relación con el paciente que se sustente en la esperanza mutua de continuidad en el tiempo y que esté basada en la confianza, respeto y responsabilidad recíproca" (Starfield, 2004, p. 166). Esto implica, además, desarrollar actividades a lo largo de todo el ciclo vital de los individuos, tanto en la atención sanitaria como en los espacios familiares, laborales y comunitarios.

- Atención integral y enfoque biopsicosocial: se requiere que el personal de salud "identifique de manera adecuada la gama de necesidades de salud de los pacientes y que disponga de los recursos para manejarlas" (Starfield, 2004, p. 197). Asimismo, esto implica que observen permanentemente los factores biológicos, psicológicos y sociales que afectan a los pacientes como parte del sistema social.

- Enfoque promocional y preventivo: se caracteriza por dos tipos de acciones. Las primeras dicen relación con acciones comunitarias, "consideradas bienes de salud pública, que deben ser implantadas a través de proyectos específicos formulados con lógica territorial o poblacional" (Ministerio de Salud, 2008, p. 86), es decir, en planes comunales y regionales de promoción de salud. Las segundas son individuales y están "dirigidas en forma específica a una persona o pequeño grupo familiar o social, que pueden ser incorporadas en planes de prestaciones individuales" (Ministerio de Salud, 2008, p. 86). 
- Trabajo en equipo: los funcionarios de la salud constituyen un equipo de trabajo integrado por profesionales y no profesionales, que debe responsabilizarse en conjunto por la salud de la familiaen y además deben vincularse con la comunidad a cargo, a través de diferentes acciones de planificación, gestión, promoción, prevención, etc. (Starfield, 2004).

- Trabajo intersectorial: la salud de las familias depende igualmente de otros sectores, además de la atención primaria; por consiguiente, éstos también deben ser integrados en el proceso.

El desarrollo y auge de este enfoque ha estado influenciado por la medicina basada en evidencia. El año 2000, Stewart $\&$ otros probaron a través de un estudio de cohorte y ensayos controlados aleatorizados que este modelo de atención era más eficiente, demostraron que reducía los exámenes diagnósticos, disminuía las visitas posteriores al médico, desincentivaba las derivaciones a especialistas y los pacientes se recuperaban más rápido y obtenían mejores resultados clínicos (M. Stewart et al., 2000). Además, Stacey \& otros realizaron en el 2014 una revisión sistemática que evidenció que cuando los pacientes están en el centro de la atención, mejoran su conocimiento sobre las pruebas diagnósticas, se sienten informados y más seguros, tienen expectativas más exactas de los efectos beneficiosos y perjudiciales de sus tratamientos y participan más en la toma de decisiones (Stacey et al., 2014). Otros estudios han demostrado que este enfoque disminuye los litigios por malas prácticas, reduce la utilización de los servicios de salud e incrementan la salud emocional y la adherencia a los tratamientos (Suárez Cuba, 2012).

No obstante, para que el sistema de salud en Chile pueda girar del modelo de atención biomédico al biopsicosocial, se ha debido implementar una serie de cambios organizacionales que permitan responder al nuevo enfoque. El "modelo ha exigido transferencia de conocimientos y habilidades entre los distintos miembros de los equipos de salud y desde y hacia los usuarios y la comunidad" (Ministerio de Salud, 2008). El problema es que la implementación de estos cambios ha ocurrido en un espacio "donde no están funcionando alineadamente los requerimientos ministeriales con la realidad que enfrentan las organizaciones que deben dar cumplimiento a estas exigencias" (Loayssa Lara, 2005), lo cual conlleva efectos negativos para quienes tienen que implementar los cambios y para quienes debieran recepcionar los beneficios de estas transformaciones.
En este sentido, uno de los factores que predice la incorporación, por parte de los implementadores, de nuevos enfoques, normas y valores organizacionales, que permite la efectividad y eficiencia de los cambios necesarios para el éxito de la nueva implementación, es el concepto de involucramiento laboral. Este concepto ha sido trabajado extensamente en países anglosajones con el fin de estudiar variables como el desempeño laboral y el compromiso organizacional y su relación con aquellas categorías como las características del trabajo y la estabilidad laboral. Para efectos de esta investigación se entendió el involucramiento laboral como el grado "en que un individuo se identifica psicológicamente con su trabajo" (Lodahl \& Kejnar, 1965, p. 333) o que "está cognitivamente preocupado, comprometido o interesado en él" (Paullay, Alliger, \& Stone-Romero, 1994, p. 225). También puede ser entendido como el grado en que el trabajo es un asunto de interés central en la vida de una persona (Dubin, 1956) o el grado en que una persona participa activamente en su trabajo y en que su autoestima se ve "afectada por su nivel de desempeño autopercibido" (French \& Kahn, 1962). En resumen, y como integra el análisis de Saleh y Hosek (1976), la literatura coincide en 4 aspectos determinantes para definir el involucramiento laboral de un individuo, en tanto éste estaría involucrado cuando: a) su trabajo es uno de los intereses centrales de su vida, b) cuando éste participa activamente en su trabajo, c) cuando éste considera que su desempeño es central para su autoestima y d) cuando éste considera que su desempeño es consistente con el concepto que tiene de sí mismo.

La no identificación con el trabajo afecta tanto la autoimagen del individuo como su desempeño en el cargo que ocupa, impactando a todo aquel que debe relacionarse con él. Esto es especialmente relevante en funcionarios como los que se desempeñan en servicios de salud, quienes representan para los usuarios del sistema el conjunto de valores y procedimientos que allí se realizan, ya que son ellos quienes establecen el contacto directo con la ciudadanía que requiere de su servicio.

En este contexto, esta investigación plantea como hipótesis descriptiva que cada uno de los siguientes factores, que conforman el involucramiento laboral, afectan el nivel de adherencia del personal de salud al nuevo modelo de atención, cuestión que en este estudio se corresponde con las variables independientes y dependiente, respectivamente: 
- Involucramiento laboral: "grado en que un individuo se identifica psicológicamente con su trabajo" (Lodahl \& Kejnar, 1965, p. 333).

- Compromiso con la organización: grado en que un individuo experimenta una cierta lealtad con la organización a la que pertenece. Junto con la lealtad, el compromiso organizacional incluye la voluntad del individuo por aumentar su esfuerzo para cumplir con las metas de la organización y el grado de alineamiento que la organización tiene con los valores de los individuos.

- Satisfacción con el trabajo: "el estado emocional resultante de la valoración del trabajo actual, pudiendo ser negativo, positivo o neutral" (Arora, 2000, p. 472). Los parámetros que se relacionan con este concepto son nueve: remuneración, promoción, supervisión, beneficios, recompensas contingentes, procedimientos operacionales, colegas de trabajo, naturaleza del trabajo y comunicación.

- Percepción del apoyo organizacional: refiere a la magnitud en que los trabajadores creen que la organización les provee del apoyo necesario, valora su contribución y se preocupa de su bienestar (Eisenberger, Fasolo, \& Davis-Lamastro, 1990). Estudios señalan que la percepción de apoyo organizacional acrecentaría el nivel de compromiso con el trabajo, al crear una atmósfera de confianza respecto de la voluntad de la organización por cumplir a cabalidad su obligación con los trabajadores.

\section{Metodología}

La estrategia de investigación utilizada fue cuantitativa, porque se colocó el énfasis en "la búsqueda de la objetividad, de las relaciones causales y de la generalización" (Canales Cerón, 2006, p. 33) de los datos. El tipo de estudio fue no experimental, porque la investigadora "no tuvo el control sobre las variables independientes y tampoco conformó grupos de estudio" (Canales Cerón, 2006, p. 33).

La unidad de análisis estuvo constituida por funcionarios profesionales y no profesionales del CESFAM Cerro Navia, el Centro de Salud Dr. Adalberto Steeger, el CESFAM Dr. Arturo Albertz y el CESFAM Lo Amor de la comuna de Cerro Navia. Se optó por esta unidad territorial porque es considerada una de las más pobres del país, situación a la que se asocian otros importantes factores de riesgo social. Según la última Encuesta CASEN 2013, cerca de un 14,6\% de los hogares de la comuna se encontraría bajo la línea de pobreza, y un 3,5\% estaría en situación de indigencia o extrema pobreza, lo que corresponde al doble de la proporción a nivel regional (Ministerio de Desarrollo Social, 2014). Los indicadores en el campo de la salud hablan de un panorama comunal de exclusión y vulnerabilidad. Se observa que la gran mayoría de la población accede al sistema previsional de salud público que pertenece a los grupos de menores ingresos A y B $(59,6 \%)$. Por otra parte, las tasas de mortalidad general $(5,1)$ y mortalidad infantil $(8,5)$ resultan sobrepasar los promedios a nivel regional y nacional, situación que deja al descubierto la precariedad de los sistemas de salud (Ministerio de Desarrollo Social, 2014).

La población está conformada por los 374 funcionarios de los centros de salud antes mencionados, ya que el nuevo modelo de atención le exige a todo el equipo de salud asumir transversalmente la responsabilidad longitudinal sobre el paciente y su familia. Entre los funcionarios hay 31 profesionales categoría A (médicos y odontólogos), 62 profesionales categoría B (asistentes sociales, enfermeras, matronas, nutricionistas, terapeutas ocupacionales, psicólogos y educadores de párvulo), 34 profesionales categoría $C$ (técnicos en enfermería de nivel superior) y 60 funcionarios categoría D, E y F (administrativos, auxiliares de servicio y chóferes)

Dado que el estudio era descriptivo-exploratorio y la información que de él se desprendió sólo puede ser aplicada a los elementos muestreados, las investigadoras no consideraron necesario utilizar cálculos estadísticos para determinar el tamaño de la muestra y sólo optaron por aplicar la encuesta al 50\% de la población. No obstante, con el objeto de evitar sesgos de selección, se aplicó el azar para determinar quiénes participaban o no de esta investigación; por tanto, podría afirmarse que la muestra es no representativa, pero sí es de naturaleza aleatoria. Es así como ésta estuvo constituida por 127 funcionarios profesionales y 60 trabajadores no profesionales de los servicios de atención primaria de la comuna de Cerro Navia.

La información se obtuvo mediante la aplicación de una encuesta, en formato papel, elaborada por las investigadoras, a partir de la literatura especializada en el tema. El instrumento estuvo constituido por 27 afirmaciones que representan las diferentes dimensiones del involucramiento laboral y se respondió sobre una escala de Likert de 4 puntos, en que 1 es "siempre", 2 es "pocas veces", 3 es "muchas veces" y 4 es "siempre". Además, este instrumento fue testeado y aplicado previamente al 10\% de la muestra. La variable dependiente y variables inde- 
CUADRO N ${ }^{\circ} 1$

VARIABLE DEPENDIENTE E INDEPENDIENTES

\section{Variable Dependiente: Adhesión al Modelo de Salud Familiar}

1. Mi trabajo en el nuevo modelo de atención familiar y comunitario es central en mi existencia

2. Las cosas que me hacen feliz en mi vida involucran mi trabajo en el nuevo modelo de atención

3. Las cosas más importantes que me pasan se relacionan con mi trabajo en el centro de salud

4. Estoy de acuerdo con el modelo de salud familiar que tiene este centro de salud

5. La mayoría de mis intereses están enfocados en implementar el nuevo modelo de salud familiar

6. Mis metas profesionales tienen que ver con el nuevo modelo de salud familiar

\begin{tabular}{|c|c|}
\hline \multicolumn{2}{|r|}{ Variables Independientes } \\
\hline \multirow{6}{*}{$\begin{array}{l}\text { Compromiso } \\
\text { con la } \\
\text { organización }\end{array}$} & 1. Estoy muy orgulloso(a) de decirles a otros que soy parte de este centro de salud. \\
\hline & 2. El pertenecer a esta institución me hace sentir importante ante mis amigos. \\
\hline & 3. Esta organización significa personalmente mucho para mí. \\
\hline & $\begin{array}{l}\text { 4. Pienso que esta es la mejor de todas las posibles instituciones de salud para } \\
\text { la cual trabajar. }\end{array}$ \\
\hline & 5. Me siento ligado(a) emocionalmente a este centro de salud. \\
\hline & 6. Estoy feliz de trabajar con el modelo de salud familiar. \\
\hline \multirow{6}{*}{$\begin{array}{l}\text { Satisfacción } \\
\text { con el trabajo }\end{array}$} & $\begin{array}{l}\text { 7. No he buscado trabajo en otro centro de salud porque estoy satisfecho con } \\
\text { mi trabajo. }\end{array}$ \\
\hline & 8. Mi institución me da seguridad laboral que no quiero perder tan fácilmente. \\
\hline & $\begin{array}{l}\text { 9. No he buscado trabajo, porque creo que debo permanecer en esta institución } \\
\text { por los beneficios económicos que tengo. }\end{array}$ \\
\hline & $\begin{array}{l}\text { 10. La capacitación y el desarrollo que he recibido en esta institución difícilmente } \\
\text { los tendría en otra. }\end{array}$ \\
\hline & $\begin{array}{l}\text { 11. Aunque el sueldo no es muy alto en esta institución, priorizo que tengo un } \\
\text { trabajo estable. }\end{array}$ \\
\hline & $\begin{array}{l}\text { 12. No he buscado trabajo en otro centro de salud, porque me interesa trabajar } \\
\text { en este lugar. }\end{array}$ \\
\hline \multirow{9}{*}{$\begin{array}{l}\text { Percepción } \\
\text { del apoyo } \\
\text { organizacional }\end{array}$} & 13. Mi jefatura directa valora mi contribución en el trabajo. \\
\hline & $\begin{array}{l}\text { 14. Mi jefatura directa tiene en cuenta mis intereses cuando toma decisiones } \\
\text { que me afectan. }\end{array}$ \\
\hline & 15. Mi jefatura directa realmente se preocupa por mi bienestar. \\
\hline & 16. La ayuda está disponible cuando tengo un problema. \\
\hline & $\begin{array}{l}\text { 17. La organización me proporciona oportunidades para desarrollarme } \\
\text { laboralmente. }\end{array}$ \\
\hline & 18. Mi jefatura directa me solicita realizar una cantidad excesiva de trabajo. \\
\hline & 19. El tiempo que se me otorga para terminar mi trabajo no es suficiente. \\
\hline & 20. Mis compañeros de trabajo se interesan por mí a nivel personal. \\
\hline & 21. Existe trabajo en equipo. \\
\hline
\end{tabular}

Fuente: Elaboración propia 
pendientes se midieron a través de las afirmaciones que se pueden observar en el cuadro $n^{\circ} 1$.

El análisis de la información se llevó a cabo a través de un proceso de análisis estadístico descriptivo bivariante, porque se estudió, tanto mediante índices descriptivos como gráficamente, la incidencia que tienen los principales factores del involucramiento laboral en la implementación de la reforma de salud. Se utilizó como variable dependiente la adhesión a la implementación del nuevo modelo de salud familiar y como variables independientes el compromiso con la organización, la satisfacción con el trabajo y la percepción del apoyo organizacional. Por último, la base de datos se organizó y manipuló con el apoyo del software de análisis de datos cuantitativos STATA que permitió ejecutar medidas de tendencia central, tablas de frecuencia, medidas de dispersión y distribución y pruebas no paramétricas.

\section{Descripción de los hallazgos}

Cuando se analiza la dimensión involucramiento laboral por separado, es posible afirmar que, en las 5 preguntas que refieren a esta variable, los sujetos se distribuyen equitativamente entre las respuestas favorables o desfavorables. Por ejemplo, frente a la aseveración mi trabajo en el centro de salud es central en mi existencia, un 22\% de los sujetos respondió que nunca estaba de acuerdo con esto, un $23,53 \%$ que pocas veces, un $36,9 \%$ que muchas veces y un $17,11 \%$ que siempre. Lo mismo sucede al momento de analizar aquellas preguntas que dicen relación con la adhesión al modelo de salud familiar. La prueba de ello es que ante la afirmación estoy de acuerdo con el modelo de salud familiar que tiene este centro de salud, un $11,23 \%$ respondió que nunca, un $40,64 \%$ que pocas veces, un $31,02 \%$ que muchas veces y un $17,11 \%$ que siempre.

En este contexto, se optó por agrupar los dos ítems de respuesta (involucramiento y adhesión al modelo de salud familiar) y aplicar la prueba no paramétrica Kruskal-Wallis que permitió apreciar que la relación que vemos en el siguiente gráfico es estadísticamente significativa. Esto se observa porque tenemos una significancia de 0,0001 , menor a 0,05 , por lo tanto, no aceptamos la hipótesis nula y concluimos que, a mayor nivel de involucramiento laboral, mayor adhesión al modelo de salud familiar, tal como se puede observar en el gráfico $\mathrm{n}^{\circ} 1$.

En la dimensión Compromiso con la Organización, se observó la misma tendencia: las personas se inclinaron equilibradamente entre actitudes favorables y desfavorables. Para ilustrar esto, frente a la afirmación estoy orgulloso(a) de decirle a otros que

\section{GRÁFICO N ${ }^{\circ} 1$ \\ RELACIÓN ENTRE EL INVOLUCRAMIENTO LABORAL Y ADHESIÓN AL MODELO DE SALUD FAMILIAR}

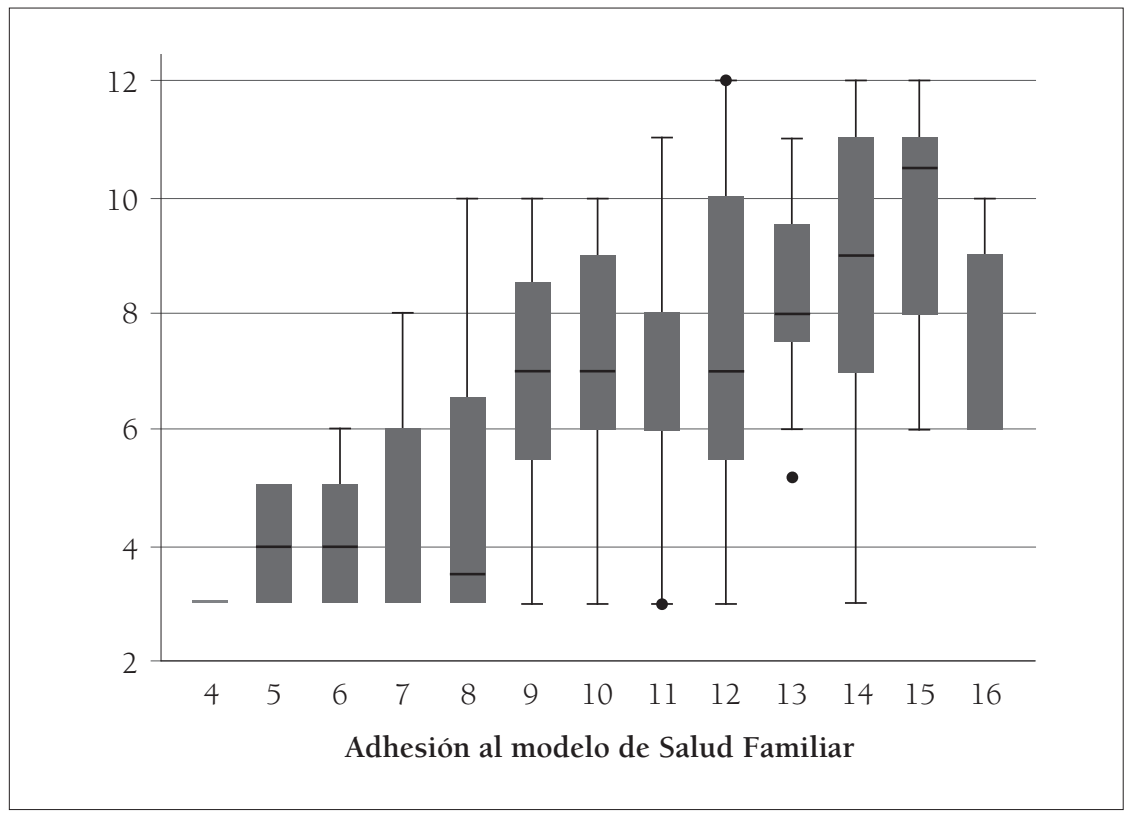

Fuente: Elaboración propia 
soy parte de este centro de salud, un $12,83 \%$ de los sujetos encuestados señaló que nunca estaba de acuerdo, un $32,62 \%$ que pocas veces, un $31,5 \%$ que muchas veces y un $22,99 \%$ que siempre. Igualmente, ante el enunciado pienso que esta es la mejor de todas las posibles instituciones de salud para la cual trabajar, un $12,83 \%$ del personal de salud manifestó que nunca estaba de acuerdo con ello, un $45,99 \%$ que pocas veces, un $27,81 \%$ que muchas veces y un $13,37 \%$ siempre. Por tanto, el grado de lealtad que experimentan los integrantes de los equipos de salud primaria de Cerro Navia con la organización en la que trabajan es neutral, puesto que sólo la mitad de ellos siente compromiso con su lugar de trabajo y la otra mitad se inclina más bien a una postura negativa.

Respecto al nivel de satisfacción fue posible apreciar una tendencia positiva. Por ejemplo, de cara a la aseveración no he buscado trabajo en otro centro de salud porque estoy satisfecho con mi trabajo, sólo un 6,42\% del personal de salud nunca está de acuerdo con esto, un $20,32 \%$ pocas veces, un $48,66 \%$ muchas veces y un $24,6 \%$ siempre. Esto se debe además a que claramente valoran la seguridad $(68,4 \%)$, los beneficios económicos $(50,8 \%)$ y la estabilidad laboral (74\%) que les otorga su trabajo. No obstante, ante la afirmación la capacitación y el desarrollo que he recibido en esta institución difícilmente la tendría en otra, la tendencia es más bien negativa, puesto que un $67,6 \%$ afirmó que nunca o pocas veces estaba de acuerdo con esto.

Por último, en el ítem Apoyo Organizacional, las respuestas en general son positivas. Muestra de ello es que ante el enunciado mi jefatura directa valora mi contribución en el trabajo, sólo un 1,6\% dijo no estar nunca de acuerdo con esta afirmación, un $17,11 \%$ pocas veces, un $44,39 \%$ muchas veces, y un $36,9 \%$ siempre. Lo mismo ocurre ante la afirmación la organización me proporciona oportunidades para desarrollarme laboralmente, pues un 5,88\% señaló no estar nunca de acuerdo con esta aseveración, un $23,53 \%$ pocas veces, un $44,39 \%$ muchas veces y $26,20 \%$ siempre.

Por otra parte, se aplicó un test no paramétrico de tendencias entre grupos desarrollado por Cuzick que produce resultados similares a Kwallis, es decir, comprueba que las proporciones de respuestas asociadas a las dimensiones de involucramiento laboral y adhesión al modelo de salud familiar sean iguales. Es así como fue posible apreciar que hay una tendencia a que las respuestas de adhesión al modelo aumenten significativamente ( $p$ valor $=0,000$ ) en la medida que aumenta el compromiso laboral, la satisfacción y el apoyo organizacional, individualmente.

\section{Discusión}

Como ha sido posible observar en los hallazgos de esta investigación, las condiciones laborales de los equipos de salud primaria son un factor relevante al momento de observar los procesos de implementación de las reformas a los sistemas de salud (Buchan, 2004), más aún cuando éstas redefinen significativamente el papel del personal que debe implementarlas. A partir de esto, es posible afirmar que el compromiso emocional con la organización, la satisfacción laboral y las condiciones de apoyo organizacional se convierten en factores críticos que impactan directamente el proceso de consumación del modelo de salud familiar en la comuna de Cerro Navia.

Los equipos de salud representan un conglomerado heterogéneo de profesionales y funcionarios que comparten y disputan esferas de interés e influencias diferenciadas frente a los cambios organizacionales (Programa de Naciones Unidas para el Desarrollo, 2009) que se llevan a cabo en sus centros de salud y de acuerdo a los hallazgos de este estudio adhieren o no a éstos conforme al grado de involucramiento laboral que sienten, por tanto, se puede deducir que también mapean sus prácticas de acuerdo a esto último.

En este sentido, resulta pertinente añadir que los equipos de salud no cumplen solo con el rol de recursos humanos de los centros de salud, sino que son -al mismo tiempo y, muchas veces, paradójicamente-implementadores de las reformas generadas en instancias superiores que, probablemente desde la difundida lógica top-down de formulación de políticas, no han considerado las particularidades de su manera de hacer las cosas (Programa de Naciones Unidas para el Desarrollo, 2009). Por tanto, es urgente que las políticas públicas consideren estas particularidades, puesto que el nivel de identificación con el trabajo, en este caso con el modelo de salud familiar, se relaciona también con el compromiso de hacerlo bien.

Es por tanto que desde el Trabajo Social emerge la necesidad de considerar la visión del rol disciplinar desde tres aspectos centrales presentes en la instalación del modelo de salud familiar en la atención primaria. En primer lugar, la participación activa que tiene el profesional en el proceso de implementación, difusión, promoción, integración de actores y adaptación de la política a la cultura organiza- 
cional y local. En segundo lugar, los trabajadores sociales en el área de la salud ejecutan actividades concretas que materializan las orientaciones técnicas que surgen desde el nivel central mediante prácticas cotidianas de intervención en lo social. $\mathrm{Y}$, en tercer lugar, destacar la gestión facilitadora realizada por estos profesionales contribuyendo a la valoración del trabajo, propiciando condiciones a favor del involucramiento laboral y la generación del cambio organizacional requerido para hacer viable la implementación del modelo de salud familiar en los centros de atención primaria.

Esto último es importante, considerando que este proceso de cambio organizacional no solo tiene relación con la estructura administrativa de los organismos de salud primaria, sino también con la red de actores que la componen. Si entendemos el rol de los trabajadores sociales como implementadores, ejecutores y facilitadores en el proceso de instalación de cambios en el modelo de salud familiar, se hace a su vez imprescindible comprender también la incertidumbre que existe frente a los desafíos y responsabilidades que deben enfrentar los profesionales para el desempeño de dichas funciones. Esto puede hacer semejanza con los planteamientos de Lipsky (2010), quien señala que las condiciones laborales de los burócratas de calle son inevitablemente inciertas, ya que éstos se encuentran confrontados cotidianamente a dilemas que no sólo son de orden ético, sino que también organizacional. En el sector de salud pública, los equipos profesionales se constituyen en torno a demandas respecto de sus servicios y desenvolvimiento laboral que suelen estar constreñidas por los recursos limitados y plazos sobre la urgencia, por tanto, las recompensas intrínsecas (apoyo, identificación con la tarea, autonomía, etc.) son las razones principales que tienen para hacer bien el trabajo.

Desde esta perspectiva, el involucramiento laboral tiene un rol crucial en la calidad de los servicios de salud, pudiendo éste promover la efectividad y productividad organizacional a partir de ganar el compromiso de los trabajadores en su trabajo y haciendo de éste una experiencia satisfactoria y con sentido (Koponen et al., 2010).

Además, los recursos laborales dispuestos por los centros de salud primaria de la comuna de Cerro Navia, como el control laboral y el apoyo social, pueden colaborar en la satisfacción de las necesidades asociadas al empleo, desde la perspectiva del trabajador, al tiempo que ayudan a enfrentar eventuales influencias negativas de las demandas del entorno, como la sobrecarga de trabajo, con el propósito de lograr las metas laborales (Koponen et al., 2010). En ese sentido, si las necesidades o las metas no son cumplidas, los individuos tienden a alejarse del trabajo, volverse apáticos y rutinarios, optando por dirigir su energía a otro ámbito.

El cambio organizacional puede resultar infructuoso $\mathrm{e}$, incluso, contraproducente para el funcionamiento de las organizaciones si éste es resistido por los trabajadores. La literatura registra de forma consensuada cómo la resistencia o aceptación al cambio por parte de los trabajadores es una de las principales causas del fracaso o del éxito de las medidas de cambio organizacional (Waldersee, Griffiths, \& Centre for Corporate Change, 1996). En base en la evidencia arrojada por esta investigación, se afirma que el involucramiento laboral, el compromiso emocional, los niveles de satisfacción laboral y la percepción de apoyo son 4 factores relevantes en las formas de reaccionar de los trabajadores frente al cambio organizacional que afecta la adhesión al modelo de atención que desea instalarse.

Sin embargo, de acuerdo al análisis expuesto por Waldersee et al. (1996), los factores asociados a las cogniciones y emociones suelen ser descuidados por los agentes a cargo de instalar el cambio organizacional al interior de los equipos, como por ejemplo los mecanismos de defensa. La emoción y el proceso emocional es relevado como una variable significativa por otro estudio de Bovey y Hede (2001) en el contexto de la resistencia al cambio organizacional, el que muestra el vínculo entre emociones (proceso emocional) y lo que definen como ideas irracionales (proceso cognitivo).

En conclusión y en concordancia con lo que nos señala la literatura especializada aspectos tales como el involucramiento laboral, el compromiso emocional, la satisfacción en el trabajo, y la percepción de apoyo organizacional determinan el éxito o fracaso de las políticas sociales y del cambio organizacional que éstas implican, tal como ha ocurrido en el proceso de implementación del nuevo modelo de salud familiar. Esto pone de manifiesto el hecho de que el éxito o fracaso de las políticas depende mucho más de los implementadores que están involucrados en el trabajo cotidiano que de las instancias centrales que las diseñan. 


\section{Bibliografía}

ARORA, R. (2000). Encyclopaedic Dictionary of Organization Behaviour (1st ed.). Delhi: Sarup \& Sons.

BOVEY, W., \& HEDE, A. (2001). Resistance to Organisational Change: The Role of Defence Mechanisms. Journal of Managerial Psychology, 16(7), 534-548. Retrieved from http://webcache.googleusercontent.com/ search?q=cache:http://www.bovey.com.au/files/rdm.pdf

BUCHAN, J. (2004). What Difference Does (good) HRM make? Human Resources for Health, 2(1), 6. http://doi. org/10.1186/1478-4491-2-6

CANALES CERÓN, M. (2006). Metodologías de Investigación Social: Introducción a los Oficios. Santiago: LOM Ediciones.

DEPARTAMENTO DE ESTADÍSTICAS E INFORMACIÓN DE SALUD. (2011). Indicadores básicos de salud. Santiago. Retrieved from http://deis.minsal.cl/ deis/indicadores/Folleto_IBS_2011.pdf

DUBIN, R. (1956). Industrial Workers' Worlds: A Study of the Central Life Interests of Industrial Workers. Social Problems, 3(3), 131-142. http://doi. org/10.2307/799133

EISENBERGER, R., FASOLO, P., \& DAVIS-LAMASTRO, V. (1990). Perceived Organizational Support and Employee Diligence, Commitment, and Innovation. Journal of Applied Psychology ght, 75(1), 51-59. Retrieved from http://citeseerx.ist.psu.edu/viewdoc/down load?doi=10.1.1.529.8528\&rep=repl \&type=pdf

FRENCH, J. R. P., \& KAHN, R. L. (1962). A Programmatic Approach to Studying the Industrial Environment and Mental Health. Journal of Social Issues, 18(3), 1-47. http://doi.org/10.1111/j.1540-4560.1962.tb00415.x

INSTITUTO NACIONAL DE ESTADÍSTICAS. (2012). Mortalidad en Chile, 2002 y 2012. Santiago. Retrieved from http://www.ine.cl/canales/menu/publicaciones/ calendario_de_publicaciones/pdf/mortalidad.pdf

KOPONEN, A. M., LAAMANEN, R., SIMONSEN-REHN, N., SUNDELL, J., BROMMELS, M., \& SUOMINEN, S. (2010). Job Involvement of Primary Healthcare Employees: Does a Service Provision Model Play a Role? Scandinavian Journal of Public Health, 38(3), 266-74. http://doi.org/10.1177/1403494810364562

LARRAÑAGA, O. (2010). Las Nuevas Políticas de Protección Social en Perspectiva Histórica. Santiago. Retrieved from http://www.ministeriodesarrollosocial. gob.cl/btca/txtcompleto/Larranaga_nvaspolitprotecsocial.pdf

LIPSKY, M. (2010). Street-Level Bureaucracy, Dilemmas of the Individual in Public Service (30th ed.). New York: The Roussel Sage Foundation.

LOAYSSA LARA, J. R. (2005). Orientación Psicosocial y Atención Familiar en Medicina de Familia: Malos Tiempos para la Lírica. Atención Primaria, 36(7), 385 387. http://doi.org/10.1157/13080305
LODAHL, T., \& KEJNAR, M. (1965). The Definition and Measurement of Job Involvement. Journal of Applied Psychology, 49(1), 24-33. http://doi.org/10.1037/ h0021692

MINISTERIO DE DESARROLLO SOCIAL. (2014). Reporte Comunal: Cerro Navia, Región Metropolitana. Santiago. Retrieved from http://observatorio. ministeriodesarrollosocial.gob.cl/indicadores/pdf/comunal_general/metropolitana/Cerro_Navia_2013.pdf

MINISTERIO DE SALUD. (2008). En el Camino a Centro de Salud Familiar. Santiago. Retrieved from http://www. bibliotecaminsal.cl/wp/wp-content/uploads/2016/03/ En-el-Camino-a-Centro-de-Salud-Familiar.pdf

MINISTERIO DE SALUD DE CHILE. (2011). ESTRATEGIA NACIONAL DE SALUD Para el cumplimiento de los Objetivos Sanitarios de la Década 2011-2020. Chemistry $\&$...

PAULlAY, I. M., ALLIGER, G. M., \& STONE-ROMERO, E. F. (1994). Construct Validation of Two Instruments Designed to Measure Job Involvement and Work Centrality. Journal of Applied Psychology, 79(2), 224-228. http://doi.org/10.1037/0021-9010.79.2.224

PROGRAMA DE NACIONES UNIDAS PARA EL DESARROLlO. (2009). La Manera de Hacer las Cosas. Santiago. Retrieved from http://desarrollohumano.cl/ idh/download/pnud_2009.pdf

SALEH, S., \& HOSEK, J. (1976). Job Involvement: Concepts and Measurements. Academy of Management Journal, 19(2), 213-224. http://doi. org/10.2307/255773

SOLAR, O., \& IRWIN, A. (2010a). A Conceptual Framework for Action on the Social Determinants of Health. Social Determinants of Health Discussion Paper 2 (Policy and Practice). Retrieved from http://apps.who. int/iris/bitstream/10665/44489/1/9789241500852_ eng.pdf?ua=1\&ua=1

SOLAR, O., \& IRWIN, A. (2010b). A Conceptual Framework for Action on the Social Determinants of Health. Social Determinants of Health Discussion Paper 2 (Policy and Practice).

STACEY, D., LÉGARÉ, F., COL, N. F., BENNETT, C. L., BARRY, M. J., EDEN, K. B., ... WU, J. H. (2014). Decision Aids for People Facing Health Treatment or Screening Decisions. (D. Stacey, Ed.)Cochrane Database of Systematic Reviews (Vol. 10). Chichester, UK: John Wiley \& Sons, Ltd. Retrieved from http://doi.wiley.com/10.1002/14651858.CD001431.pub4

STARFIELD, B. (2004). Atención Primaria. Equilibrio entre Necesidades de Salud, Servicios y Tecnología. Barcelona: Masson-PALTEX.

STEWART, M. (2003). Patient-centered Medicine: Transforming the Clinical Method (2st ed.). Abingdon: Radcliffe Medical Press. 
STEWART, M., BROWN, J. B., DONNER, A., MCWHINNEY, I. R., OATES, J., WESTON, W. W., \& JORDAN, J. (2000). The Impact of Patient-Centered Care on Outcomes. The Journal of Family Practice, 49(9), 796-804. Retrieved from http://www.ncbi.nlm.nih. gov/pubmed/11032203

SUAREZ CUBA, M. Á. (2012). Medicina Centrada en el Paciente. Revista Médica La Paz, 18(1), 67-72.

VÉLIZ, X. V. (2012). ¿Hacia o en la segunda transición demográfica? Los cambios poblacionales de Chile desde un enfoque de género. Anales, 3, 105-125. Retrieved from http://www.anales.uchile.cl/index.php/ ANUC/article/viewFile/21731/23041

WALDERSEE, R., GRIFFITHS, A., \& CENTRE FOR CORPORATE CHANGE. (1996). The Changing Face of Organisational Change. Sidney: Center for Corporate Change, Australian Graduate School of Management University of New South Wales. 